\title{
Berinjela (Solanum melongena L.) - mito ou realidade no combate as dislipidemias?
}

\author{
Maria da Conceição R. Gonçalves*, Margareth de F.F. Melo Diniz, José Damião C. Borba, \\ Xirley P. Nunes, José M. Barbosa-Filho
}

Laboratório de Tecnologia Farmacêutica “Delby Fernandes de Medeiros”, Universidade Federal da Paraíba, Caixa Postal 5009, 58051-970, João Pessoa, PB, Brasil

\begin{abstract}
RESUMO: A dislipidemia é considerada um dos fatores de risco mais importantes para o desenvolvimento da doença arterial coronariana. Como resultado, tratamentos efetivos com fármacos foram desenvolvidos para combatê-la, porém, apresentam alto custo e efeitos colaterais. Considerando este fato, pacientes têm recorrido a tratamentos utilizando alimentos conhecidos científica e popularmente por possuírem efeito hipolipemiante. Várias pesquisas vêm sendo realizadas com esses alimentos como a berinjela no sentido de investigarem seus possíveis efeitos na redução dos lipídios sangüíneos. A berinjela (Solanum melongena L.) tem sido alvo desses estudos devido a sua utilização popular para dietas de emagrecimento, por aumentar a eliminação de gorduras e combater o excesso de colesterol. Este trabalho teve como objetivo fazer uma revisão da literatura científica sobre a espécie Solanum melongena (berinjela) da família Solanaceae, associado a redução de gorduras no organismo. Para este fim foram consultados vinte e cinco referências.
\end{abstract}

Unitermos: Solanum melongena, berinjela, dislipidemia, colesterol, revisão.

\begin{abstract}
Eggplant (Solanum melongena L.) - myth or reality in the fight against the dislipidemy?". High cholesterol is considered one of the most important factors for the coronary arterial disease development. As a result, effective treatments with medicine were developed to fight it; even so, they present high cost and collateral effects. Considering this fact, patients have required treatments using popular and scientific wellknown food whose effect is to decrease the fat rate. Several researches are being carried out with eggplant with the objective of investigating their possible effects in the reduction of the blood fat. The species Solanum melongena has been the target of several studies due to its popular use in weight-loss diets, as it increases the elimination of fat and fights the exceeding cholesterol. This work aims at showing a literature review about the species Solanum melongena, of the family Solanaceae, associated with the reduction of fats by the organism. For this purpose twenty-five references were consulted.
\end{abstract}

Keywords: Solanum melongena, eggplant, dislipidemy, cholesterol, review.

\section{INTRODUÇÃO}

Segundo a Organização Mundial de Saúde, as doenças cardiovasculares apresentam uma incidência elevada em todo o mundo e têm como fator de risco as dislipidemias que, isoladamente ou associadas a outros fatores como tabagismo, obesidade, diabetes, etc. podem com o avançar da idade, provocar o aparecimento dessas patologias (III Diretrizes sobre dislipidemias e ateriosclerose, 2001). Embora muitos progressos tenham contribuído para melhor entendimento do seu mecanismo, ainda permanece muito a ser desvendado e eventos por ela desencadeados como infarto do miocárdio, por exemplo, continuam sendo causa de óbitos nas sociedades urbanas.

Estudos de intervenção têm mostrado que o risco para a doença arterial coronariana pode ser reduzido, quando os níveis séricos de colesterol são controlados pela dieta ou tratamento farmacológico. Atualmente, existem muitos tipos de medicamentos capazes de reduzir as hiperlipidemias, sendo os mais utilizados as estatinas e os fibratos. Porém, se este tratamento é eficiente, também é caro e não isento de efeitos colaterais, proporcionais à dose do medicamento utilizado.

Considerando este fato, os pacientes têm recorrido a tratamentos alternativos para o controle da dislipedimias através de alimentos ricos em fibras. Para os médicos naturalistas, a berinjela, a cenoura, a cebola e o jiló são os vegetais mais indicados para reduzir o colesterol sérico (Balbach; Boarim, 1992).

A utilização de plantas com fins medicinais vem contribuindo como um vasto campo de pesquisa que fornecerá importantes subsídios geradores de mudanças na sistematização da assistência e do ensino da saúde, que deverão refletir na identificação e no preparo de plantas medicinais valiosas, além do seu uso apropriado quanto à posologia, indicação, limitações e riscos de utilização. Um exemplo disto é à busca de novos fitoterápicos, para minimizar os efeitos colaterais e os altos custos do tratamento medicamentoso. 


\section{Dislipidemias: definição, causas e conseqüências}

Existe um delicado equilíbrio no organismo para cobrir as necessidades de lipídios das células e, ao mesmo tempo, evitar seu acúmulo excessivo. Por razões não muitos claras, esse equilíbrio pode romper-se, elevando o nível de um ou mais componentes lipídicos na corrente sangüínea, ao que se denomina dislipidemia. As alterações fisiopatológicas envolvidas na sua gênese promovem o acúmulo da Lipoproteína de Baixa Densidade (LDL-CL) e da Lipoproteína de Muito Baixa Densidade (VLDL-CL) nos compartimentos plasmáticos, resultando em Hipercolesterolemia e Hipertrigliceridemia, respectivamente. $\mathrm{O}$ diagnóstico precoce e a redução dos níveis séricos desses componentes, quando elevados, têm contribuído para sensível queda da incidência e da mortalidade por doença cardiovascular (Mahan; EscottStump, 2004).

De acordo com a classificação etiológica, a dislipidemia pode ser: Dislipidemia Primária conseqüente às causas genéticas e/ou ambientais, dentre as quais se destacam: Hipercolesterolemia familiar heterozigótica - evidenciada desde o momento do nascimento; Hipercolesterolemia familiar combinada - manifesta-se com o aumento das concentrações plasmáticas de colesterol e triglicérides, nem sempre simultaneamente e Hipercolesterolemia poligênica forma mais comum, com intervenção de genes distintos, os quais promovem pequeno aumento das concentrações de LDL-CL. Dislipidemias Secundárias - compreendem aquelas relacionadas às doenças: Diabetes Melittus tipo II, Hipotireoidismo, Síndrome Nefrótica, Insuficiência Renal Crônica, Hepatopatias Colestáticas Crônicas, Obesidade etc; assim como às doenças secundárias e aos efeitos de medicamentos (anti-hipertensivos, corticóides, inibidores de proteases) e aos hábitos de vida inadequados (tabagismo, etilismo).

$\mathrm{O}$ interesse no estudo dos lipídios surgiu no século XIX com Vogel em 1847, que foi o primeiro investigador a detectar a presença de colesterol nas placas de ateroma. No início do século $\mathrm{XX}$, na Alemanha, estudos experimentais realizados com ratos demonstraram que estes, quando alimentados com dieta rica em colesterol, desenvolviam hipercolesterolemia e lesões ateroscleróticas (Bertolami; Bertolami 1986).

Nas últimas décadas, a relação lipídios e lipoproteínas e a doença arterial coronariana (DAC) tem sido intensamente estudada. A procura continuada do entendimento de como as dislipidemias é capaz de levar a, ou facilitar o desencadeamento da aterosclerose, vem fascinando muitos investigadores, suscitando em importantes descobertas.

Dentre as patologias pertencentes ao grupo de doenças do aparelho circulatório, a doença arterial coronariana tem sido considerada problema de saúde pública, na Europa e América do Norte, desde o começo do século. Atualmente, no Brasil, também é considerado problema de saúde pública, tendo em vista o seu papel no perfil de mortalidade e as alterações patológicas que acarretam muitas vezes irreversíveis, com conseqüente incapacidade residual.

Amplas pesquisas epidemiológicas e experimentais realizadas nas últimas décadas estabeleceram os principais fatores de risco para a doença arterial coronariana conseqüente à aterosclerose. Entre elas, destacam-se, pela força de correlação com a DAC: tabagismo, hipertensão arterial, hipercolesterolemia, HDL-C baixo, Diabetes Mellitus, presença de hipertrofia ventricular esquerda, obesidade, história familiar positiva, mulheres na menopausa sem o uso de reposição hormonal (Rackley, 1997), estresse, sedentarismo e alguns hábitos relacionados ao estilo de vida como dieta rica em calorias, gorduras saturadas, colesterol, sal e consumo de bebidas alcoólicas. Tais fatores mostraram-se independentes, variando, entretanto, a associação entre eles de acordo com as amostras populacionais em observação.

A doença arterial coronariana é a doença cardiovascular mais mortal, sendo a principal causa de morte em homens com idade acima de 45 anos e mulheres com idade acima de 65 na Europa. No Brasil, a prevalência de doenças crônico-degenerativas quase duplicou entre as décadas de 60 e 70. No período entre 1940-1980, a mortalidade por enfermidades cardiovasculares, que correspondia a $10 \%$ na década de 30 , elevou-se para $35 \%$ em 1980. Acredita-se que a doença coronariana seja responsável por mais de $35 \%$ dos óbitos anuais brasileiros, sendo que em $40 \%$ desses casos, as vítimas apresentam mais de 40 anos de idade (World Health Statistics Annual, 1995).

Investigações anatomopatológicas, epidemiológicas, clínicas e terapêuticas demonstraram suficiente e exaustivamente a vinculação entre hipercolesterolemia e DAC. Por outro lado, a redução dos níveis plasmáticos de colesterol total e, particularmente, da fração LDL-C, quer através de modificações no estilo de vida, quer através de medicamentos, tem-se mostrado eficaz para a diminuição de eventos coronarianos, normalização da função endotelial, estabilização e, até mesmo, regressão de lesões ateroscleróticas. Como resultado, tentativas para diminuir esta doença são de extrema importância, e a redução na sua ocorrência e progressão continuam sendo importantes metas na medicina preventiva.

\section{Berinjela (Solanum melongena): alimento hipolipemiante}

Nas primeiras décadas do século $\mathrm{XX}$, foi confirmado que a berinjela (Solanum melongena L.) comum, escura, ingerida liquefeita crua e com casca é muito eficiente nesse particular, cabendo a Roffo (1943), na Argentina, demonstrar pela primeira vez, em animais (coelhos e cães) e humanos, que esse vegetal tem a virtude de diminuir o colesterol e reduzir a ação das gorduras 


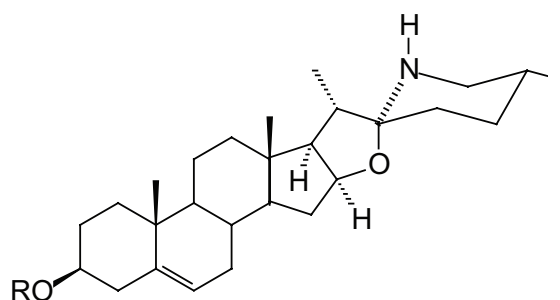

Solamargina

$\mathrm{R}=$ Rham-Rham-Glu

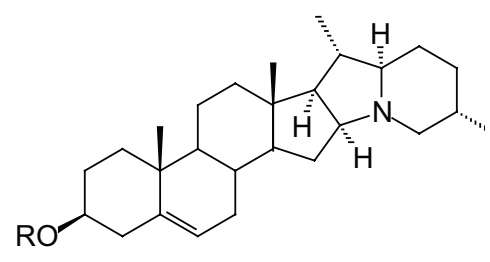<smiles>CCCCC1(NCCC)OC2C(CC3C4CC=C5CC(O)CCC5(C)C4CCC32C)C1C</smiles>

Solanina

$\mathrm{R}=$ Rham-Gal-Glu
Solasodina

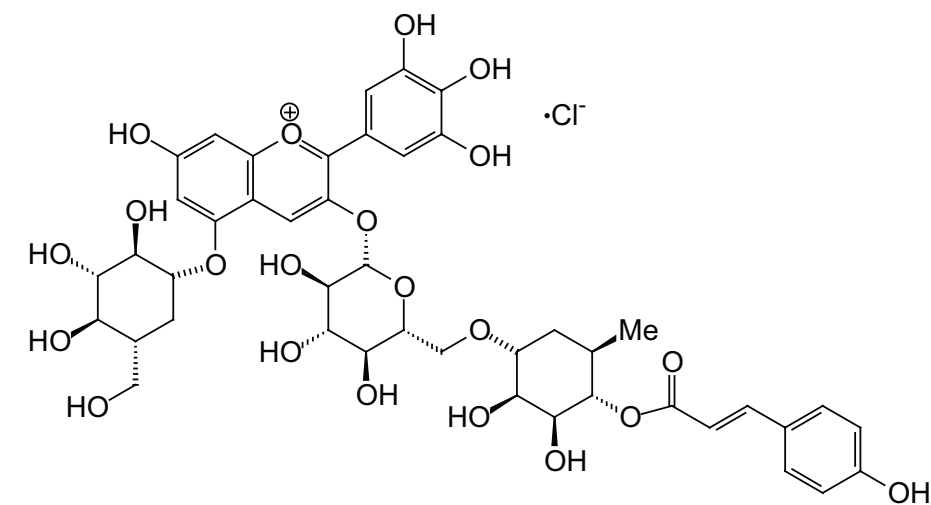

Nasunina<smiles></smiles>

\section{Delfinidina}

Figura 1. Constituintes químicos de Solanum melongena, associados a redução de gorduras no organismo

sobre o fígado.

Trabalhando com colesterol marcado com carbono radiotivo, Kritchevsky et al. (1975), concluíram que o chá de berinjela diminui o colesterol sérico em coelhos, pelo efeito de diminuição da absorção do colesterol da dieta.

Já o trabalho de Kusano et al. (1987) demonstrou que os alcalóides das solanáceas, solamargina, solanina e solasodina (Figura 1), também encontrados em S. melongena seriam capazes de inibir a conversão enzimática de diidrolanosterol em colesterol, agindo num ponto além daquele em que age a enzima HMG-CoAredutase, no metabolismo deste lipídio.

Kayamori e Igarashi (1994) testaram o efeito da nasunina, uma antociana extraída da berinjela, e da delfinidina (sua aglicona) (Figura 1) nos níveis séricos de colesterol em ratos, concluindo que ambas as substâncias contribuem para a redução e para a elevação do colesterol sérico total e do colesterol HDL, respectivamente, o que se deve, em parte, à inibição da absorção intestinal de colesterol e ácidos biliares.

Em 1995, Urrea et al., demonstraram que o extrato alcoólico do fruto de S. melongena apresentava atividade hipocolesterolemiante significativa em ratas. Mediante um estudo histopatológico da aorta se estabeleceu este efeito da dieta e do tratamento sobre a lesão aterosclerótica.

Em 1998, Cruz et al. estudando 20 pacientes hipertensos, com idade média de 59,6 anos, Índice de Massa Corporal (IMC) de 28,1 kg/m², por 222 dias de observação, usando o suco de berinjela violeta escura com casca liquefeito com suco de laranja, obtiveram uma redução de até $30 \%$ no colesterol total e $43 \%$ na fração LDL-colesterol, sem alteração significativa das frações HDL-CL e VLDL-CL, bem como do peso corporal.

No estudo de Ribeiro-Jorge et al. (1998) foi avaliado o efeito do suco da berinjela sobre os lipídeos plasmáticos, o colesterol tecidual e a peroxidação lipídica em coelhos hipercolesterolêmicos. Nos animais hipercolesterolêmicos alimentados com ração acrescentada de colesterol, gordura de babaçu e suco de berinjela observou-se redução significante do colesterol total plasmático em 19\% quando comparado ao grupo hipercolesterolêmico que fez uso da mesma 
ração suprimindo-se o suco de berinjela. Os níveis de VLDL-CL e HDL-CL não apresentaram modificações significantes, o colesterol LDL reduziu-se em $29 \%$ e os triglicerídeos em $38 \%$. Houve redução do peso corpóreo que foi interpretado como conseqüência do elevado teor de fibras encontrado na berinjela.

Flavonóides isolados de $S$. melongena mostraram atividade antioxidante potente. Concentração de malondialdeido, hidroperoxidase e conjugados estavam significativamente diminuídos. A atividade da catalase foi encontrada aumentada significativamente nos tecidos normais e com colesterol em ratos alimentados com $1 \mathrm{mg}$ de flavonóides de berinjela. A concentração de glutationa também se mostrou elevada nos animais do experimento. Os elevados níveis de glutationa e ação da catalase estimulada significativamente pode ser responsável pelo efeito antioxidante desses flavonóides (Sudheesh et al., 1999).

Silva et al. (1999) testaram o efeito do chá de berinjela ( $S$. melongena) nos níveis séricos e hepáticos de colesterol e triglicérides em ratos adultos. 36 ratos Fisher foram divididos em três grupos. Após 28 dias foram sacrificados e dosaram-se os níveis de colesterol e triglicérides séricos e hepáticos. Os resultados obtidos indicaram que, nas condições experimentais utilizadas, o chá de berinjela eleva o colesterol sérico, reduz o hepático e tem pouco ou nenhum efeito sobre os triglicérides, tanto séricos quanto hepáticos.

No ano seguinte, Guimarães et al. (2000) observaram o efeito do chá de berinjela em 38 pacientes voluntários com hipercolesterolemia por cinco semanas. O colesterol total e frações, triglicérides e apolipoproteínas A e B foram medidos no sangue durante o experimento (Início, 03 semanas e 05 semanas). Os resultados sugerem que o infuso de $S$. melongena tem um modesto e transitório efeito na diminuição do colestrerol total, fração LDL-CL e apolipoproteína B. Não sendo diferente pelos resultados obtidos com a dieta padrão para hipercolesterolemia e a pratica da atividade física.

Veiga et al. (2000) estudaram o extrato bruto aquoso de $S$. melongena (berinjela) na diminuição do colesterol plasmático de ratos, tratados por quinze dias de injeção oral $(0,5 \mathrm{~g} / \mathrm{mL}, 0,2-0,4 \mathrm{~mL})$ ou ingestão voluntária $(0,2 \mathrm{~g} / \mathrm{mL})$ com este extrato bruto aquoso, verificaram que houve uma diminuição significativa de colesterol nos animais.

Cintra et al. (2000) estudaram a atividade hipocolesterolêmica e toxicidade em fase de tratamento sub-crônico do extrato hidroalcoólico de S. melongena em ratos. $\mathrm{O}$ extrato hidroalcoólico foi administrado na dose de $180 \mathrm{mg} / \mathrm{kg}$ (v.o) durante 30 dias em 10 ratos Wistar. Os parâmetros observados foram: ingestão de água, consumo de ração, diurese, desenvolvimento ponderal, análise hemato-bioquímica e histopatologia. Os resultados mostraram que os animais não apresentaram diferença significativa em todos os termos observados, com exceção da análise histopatológica onde foi observado $100 \%$ de necrose focal de hepatócitos.

Em estudos realizados, para avaliar o efeito da administração de $S$. melongena no metabolismo do colesterol aterogênese em camundongos deficientes para o receptor de LDL, Enéas et. al. (2002) demonstraram que os animais submetidos à dieta aterogênica e à berinjela evidenciou-se um aumento do colesterol sérico total e nas frações aterogênicas, mostrando um efeito hipercolesterolêmico comparado ao grupo controle.

Em 2004, Odetola et al. estudaram o efeito hipolipemiante de frutas frescas e maduras de Solanum melongena e Solanum gilo em coelhos. A estes animais foi induzido um aumento de colesterol, tornando-os hipercolesterolêmicos, aos quais foi administrada uma alimentação contendo $10 \%$ de cada fruta por 6 (seis) semanas. Comparados ao grupo controle observaram que houve uma redução dos níveis séricos de colesterol total, triglicerídeos e fração LDL-CL e um aumento da fração HDL-CL. S. melongena e $S$. gilo reduziram significativamente e respectivamente os níveis de colesterol total de 65,4 e 52,7\%, triglicerídeos de 47,7 e $27 \%$, LDL-CL de 85 e $83 \%$. E um aumento de 24,7 e $25 \%$, respectivamente de HDL-CL. Concluindo portanto que $S$. melongena e $S$. gilo têm forte efeito hipolipemiante, podendo serem utilizadas no tratamento de doenças associadas com o aumento do colesterol, corroborando os estudos de Matos et al. (2005) que estudaram em ratos uma dieta modelo capaz de induzir hipercolesterolemia, sem afetar as funções hepáticas e o desenvolvimento dos animais.

Botelho et al. (2004) avaliaram o efeito do extrato de berinjela no metabolismo de colesterol e na arterogenesis em LDLR (-/-) em ratos. Os animais em número de 17 foram alimentados com uma dieta padrão e 21 animais com dieta arterogênica, durante 12 semanas. Ao grupo controle foi administrado água e aos demais, extrato de berinjela. Fígado, soro, lipídios fecais e lipoproteínas foram analisados. Foi avaliada também a tensão oxidativa. Foi observado que o extrato de berinjela não diminuiu o colesterol nem afetou as lipoproteínas nos animais com arteriosclerose. Como também não diminuiu o colesterol no protoplasma, nem preveniu o desenvolvimento de arteriosclerose em LDLR (-/-) nos animais. Surpreendentemente, a berinjela aumentou a oxidação, que representou um fator de risco para a arteriosclerose. Com estes resultados os autores concluíram que o extrato de berinjela não deve ser considerado um agente hipolipemiante.

No mesmo ano de 2004, Silva et al. estudando o extrato de berinjela, em cápsula, comercializado no Brasil, em pacientes com dislipidemia, através de um estudo duplo cego randomizado, onde 41 pacientes, sendo 21 experimentos e 20 placebos, receberão cápsulas contendo $450 \mathrm{mg}$ de extrato de berinjela, para ser utilizado duas vezes ao dia durante três meses. Observaram que não houve diferença significativa entre o grupo experimental e o placebo, não obtendo resposta quanto à diminuição 
das taxas de colesterol e suas frações, assim como nas taxas de triglicerídeos. Concluíram então que, a cápsula de berinjela comercializada no Brasil, não tem efeito hipolipemiante, corroborando o trabalho de Praça et al. (2004), ao comparar o uso do suco da berinjela, em pacientes com dislipidemias, concluindo também não haver diminuição nos níveis de colesterol desses pacientes.

No entanto, em 2005 Jenkins et al. em um estudo comparativo com pacientes usando a medicação Statin ${ }^{\circledR}$ (lovastatina) mais dieta pobre em gorduras saturadas, com um grupo usando uma pasta confeccionada com alimentos funcionais contendo berinjela, leite e carne de soja, amêndoas, quiabo, aveia, Psyllium e cevada e grupo controle (dieta pobre em gordura saturada) encontraram no sangue dessas pacientes níveis de colesterol diminuído em $33,3 \pm 1,9 \%, 29,6 \pm 1,3 \%$ e 8,5 $\pm 1,9 \%$ respectivamente, depois de 4 (quatro) semanas de experimento. Concluindo que a pasta confeccionada com alimentos funcionais podem não possuir a potencia da medicação lovastatina, porém representa uma abertura para novos estudos e também uma opção terapêutica viável para a prevenção primária de pacientes portadores de doenças cardiovasculares, incluso colesterol aumentado.

\section{CONCLUSÃO}

Por tudo isto, concluí-se que os resultados de pesquisas realizadas com a espécie Solanum melongena L. (berinjela) são de extrema importância para a redução da dislipidemia, em especial a hipercolesterolemia quando esta foi utilizada na forma de suco do fruto com casca. Todavia em alguns estudos citados nesta revisão é demonstrado que se faz necessário um aprofundamento maior sobre a sua eficácia, para que seus mitos e verdades venham a ser desvendados, comprovando seus benefícios sem causar danos à população que passe a utilizá-la, cabendo aos futuros pesquisadores este desafio.

\section{AGRADECIMENTOS}

Os autores agradecem ao Banco de Dados NAPRALERT da University of Illinois, Chicago, U.S.A., pelo levantamento bibliográfico da espécie Solanum melongena. J.M. Barbosa-Filho agradece ao CNPq/Brasil pela Bolsa de Pesquisa e apoio financeiro.

\section{REFERÊNCIAS}

Balbach A, Boarim DSF 1992. As Hortaliças na Medicina Natural. $\quad 2^{\text {a }}$ edição. Edições Vida Plena. Itaquaquecetuba-SP.

Bertolami MC, Bertolami V 1986. A hipercolesterolemia e as demais hiperlipidemias. Rev Bras Med 43: 112-121.

Botelho FV, Enéas LR, Cesar GC, Bizzotto CS, Tavares E, Oliveira FA, Gloria MB, Silvestre MP, Arantes RM, Alvarez-Leite JI 2004. Effects of eggplant (Solanum melongena) on the atherogenesis and oxidative stress in LDL receptor knock out mice (LDLR(-/-). Food Chem Toxicol 42: 1259-1267.

Cintra DEC, Carvalho JCT, Caputo LRG, Vicita WA 2000. Atividade hipocolesterolêmica e toxicidade em fase de tratamento sub-crônico do extrato hidroalcólico de Solanum melongena (Berinjela). Farmacotécnica e controle de qualidade. Anais do XV Reunião Atualidade de Sociedade de Biologia Experimental.

Cruz J, Cruz HMM, Barbosa-Filho JM 1998. Tratamento das Hiperlipidemias. In: Jenner Cruz, Rui Toledo Barros, Helga Maria Mazzarolo Cruz (Org.). Atualidades em Nefrologia 5. Ed. Sarvier. São Paulo v. 5, p. 460-467.

III Diretrizes Brasileiras sobre Dislipidemias e Aterosclerose 2001. Publicação do Departamento de Aterosclerose da Sociedade Brasileira de Cardiologia. Data final de elaboração da versão: 23 de agosto de 2001.

Enéas LR, Alvarez-Leite J, Botelho FV, César GC, Arantes RME 2002. Berinjela (Solanum melongena) aumenta fatores de risco de aterosclerose em modelo de hipercolesterolemia familiar. Anais da IX Semana de Iniciação Científica da Universidade Federal de Minas Gerais.

Guimaraes PR, Galvão AMP, Batista CM, Azevedo GS, Oliveira RD, Lamounier RP, Freire N, Barros AMD, Sakurai E, Oliveira JP, Vieira EC, Alvarez-Leite JI 2000. Eggplant (Solanum melongena) infusion has a modest and transitory effect on hypercholesterolemic subjects. Bras J Med Biol Res 33: 1027-1036.

Jenkins DJA, Kendall CWC, Marchie A, Faulkner DA, Wong JMW, Souza R, Emam A, Parker TL, Vidgen E, Trautwein EA, Lapsley KG, Josse RG, Leiter LA, Singer W, Connelly PW 2005. Direct comparison of a dietary portfolio of cholesterol-lowering foods with a statin in hypercholesterolemic participants. Am J Clin Nutr 81: 380-387.

Kayamori F, Igarashi K 1994. Effects of dietary nasunin on the serum cholesterol level in rats. Biosci Biotech Biochem 58: 570-571.

Kritchevsky D, Tepper AS, Story JA 1975. Influence of an eggplant (Solanum melongena) preparation on cholesterol metabolism in rats. Exp Pathol Jena 10: 180-183.

Kusano G, Takahashi A, Nozoe S, Sonoda Y, Sato Y 1987. Solanum alkaloids as inhibitors of enzmatic conversion of dihydrolanosterol into colesterol. Chem Pharm Bull 35: 4321-4323.

Mahan LK, Escott-Stump S 2004. Krause's Food, Nutrition and Diet Therapy. 11e. Singapore, Elsevier.

Matos SL, Paula H, Pedrosa ML, Santos RC, Oliveira EL, Chianca-Junior DA, Silva ME 2005. Dietary models for inducing hypercholesterolemia in rats. Braz Arch Biol Technol 48: 203-209.

Odetola AA, Iranloye YO, Akinloye O. 2004. Hypolipidaemic potentiais of Solanum melongena and Solanum gilo on hypercholesterolemic rabbits. Pakistan Journal of Nutrition 3: 180-187.

Praça JM, Thomaz A, Caramelli B 2004. Eggplant (Solanum melongena) extract does not alter serum lipid levels. Arq Bras Cardiol 82: 269-76.

Rackley CEMD 1997. Hypercholesterolemia. Drugs of Today 33: 307-314.

Ribeiro-Jorge PAR, Neyra LC, Osaki RM, Almeida E, Bragagnolo N 1998. Efeito da berinjela sobre os 
lípides plasmáticos, a peroxidação lipídica e a reversão da disfunção endotelial na hipercolesterolemia experimental. Arq Bras Cardiol 70: 87-91.

Roffo AH 1943. Eggplant in decholesterolization (Solanum melongena) Bol Inst Med Exp Estud Trat Câncer 20: 515.

Silva M, Santos RC, O’leary MC, Santos RS 1999. Effect of aubergiene (Solanum melongena) on serum and hepatic cholesterol and triglycerides in rats. Braz Arch Biol. Technol 42: 339-342.

Silva, GEC, Takahashi MH, Eik-Filho W, Albino CC, Tasim GE, Serri LAF, Assef AH, Cortez DAG, Bazotte RB 2004. Ausência de efeito hipolipemiante da Solanum melongena L. (Berinjela) em pacientes hiperlipidêmicos. Arq Bras Endocrinol Metabol 48: 368-373.

Sudheesh S, Sandhya C, Koshy S, Vijayalaskshmi NR 1999. Antioxidant sctivity of flavonoids from Solanum melongena. Phytother Res $13: 393-396$.

Urrea ADL, Solanum melongena . Rev Comb Cienc Quim Farm 23: 13-24.

Veiga MAL, Utida L, Crespigio J, Nascimento M, Nisioka $\mathrm{R}$, Rodrigues $\mathrm{T}$, Yamada $\mathrm{M}$, Aoki $\mathrm{S}$, Nishibe $\mathrm{S}$, Chiarelli L, Santos F, Siqueira L, Teixaira O, Zaia DAM, Zaia CTBV 2000. Extrato bruto aquoso de Solanum melongena (Berinjela) diminui o colesterol plasmático de ratos. XV Reunião Anual da Federação de Sociedades de Biologia Experimental. Caxambu, Brasil.

World Health Statistics Annual 1995. Edition, World Health Organization, Geneva 1996. 